\title{
An Exploration of the Burnout Situation on Teachers in Two Schools in Macau
}

\author{
Andrew L. Luk • Bessie P. S. Chan - Selwyne W. Cheong • \\ Stanley K. K. Ko
}

Accepted: 14 March 2009/Published online: 2 October 2009

(C) The Author(s) 2009. This article is published with open access at Springerlink.com

\begin{abstract}
To explore the burnout situation of teachers in two schools in Macau and to investigate any differences made of demographic characteristics. The relation of burnout on social problem solving and holistic health of teachers is also studied. A self reported questionnaire with the Chinese Maslach Burnout Inventory (C-MBI), Chinese Social Problem Solving Inventory (C-SPSI) and the Body-Mind-Spirit Well-Being Inventory (BMSWBI) were used. Totally 138 teachers participated in the study. With Hong Kong data as a reference, Macau teachers scored in the average range of burnout in emotional exhaustion and depersonalization but scored in the low range of burnout in personal accomplishment. Significance differences were found in some dimensions of burnout in age, marital status, teaching experiences, education background and satisfaction with income. Both C-SPSI and BMSWBI were found to be significantly negative correlated with emotional exhaustion and depersonalization and positive correlated with personal achievement. The findings identified those with greater degree of burnout, to whom more attention should be paid. The study also contributed to the limited literature on the quality of life of teachers in Macau. Finally, the findings added on the data base for comparison internationally among the Chinese population.
\end{abstract}

Keywords Burnout - Demographic - Macau teachers · MBI - Problem solving · Holistic health

\section{Introduction}

Macau is a city near Hong Kong and famous for its gaming business. After the government opened the gaming licensure in 2002, both the number of casinos and the revenue generated from the gaming business increase. People working in casinos can usually receive nearly double the pay of those with the same education background but working in other sectors. It attracts quite a number of young people to work there especially those with less

A. L. Luk $(\bowtie) \cdot$ B. P. S. Chan · S. W. Cheong · S. K. K. Ko

Kiang Wu Nursing College of Macau, Macau, SAR China

e-mail: luk@kwnc.edu.mo 
motivation to study. The prevailing atmosphere of working in casinos is so great that it affects the traditional Chinese values of studying hard in order to earn more money for a better life. Community voices increasing concerns about schools enhancing teaching of ideals, will power and personal values so that young people can be positively developed and better prepared to resist the temptation of gambling in the city.

In Macau, there is a 15-year free non-tertiary education system with direct promotion from primary to secondary school without any public examination. There is no public examination either but individual admission examination after secondary school education for entry into local universities. The total number of schools is 82 with 5,100 teachers and the overall teacher-student ratio is 1:17.7 (Education and Youth Affairs Bureau 2008a). As a statutory requirement, teachers who are qualified to teach in kindergarten, primary and secondary school must be graduates from the school of education. Those who are not from the teaching background have to complete a teaching course and pass an examination organized by the government in order to obtain professional qualification in teaching (Education and Youth Affairs Bureau 2008b). As a Chinese community, people are expected to behave in line with their social roles. At schools, teachers are expected to be knowledgeable and with high moral standard. Students are to be well-disciplined, self-engaged and reflective in learning. Teachers are dominant and directive in the Eastern pedagogical culture (Zhang 2007). Therefore, teaching has long been a respectable profession in the Chinese culture. However, with the increasing demand from the public to meet the knowledge needed by the changing world and the greater effort for moral education at the same time, teaching is becoming more stressful and teachers are more prone to burnout.

In the prevention of burnout of teachers, a stress reduction program was designed for one primary and one secondary school in Macau as requested from the school principals. These two schools were established in 2002 by a charity organization to receive students, mainly new immigrants from Mainland China. They are non-religious, non-profit making private schools with full tuition sponsored by the government. Since no formal study on burnout was conducted on this group of teachers, it is important to get to know their burnout situation before the implementation of the stress reduction program. This study is to explore the burnout situation of the teachers of these two schools. Furthermore, this study also examines the effect of burnout related to demographic variables, their problem solving ability and general well being.

The term "burnout" was first coined by Freudenberger (1974) to characterize a situation experienced by human service professionals who appear to be exhausted or up to a state of inability to perform their tasks effectively or sometimes even to care for their clients. Maslach (1976) defined burnout as a condition in which one loses all concerns, feeling toward the person one works with and comes to treat them as impersonal objects. Maslach and Jackson (1981) developed a multidimensional model to delineate three aspects of the content of burnout, namely: (1) emotional exhaustion (feeling of drain and tiredness), (2) depersonalization (treating clients as impersonal objects), and (3) lack of personal accomplishment (feeling of inefficiency, ineffectiveness and inadequacy). Since Maslach and Jackson's model has been adopted widely in investigating teachers' burnout syndromes and the Maslach Burnout Inventory (MBI) is also consistently found to be a reliable instrument, this model was adopted in the present study (Lau et al. 2005; Mo 1991; Sarros and Sarros 1990; Xu et al. 2004). Burnout has generally been viewed as a failure to cope with job stress and there is an international concern about stress and burnout among school teachers (Dworkin 1997; Maslach and Jackson 1981). Numerous studies have been conducted to investigate sources of stress of school teachers and its management. Broadly speaking, job nature in terms of overwork, classroom discipline, lack of parental support; 
personal factor consisting of blaming oneself, low self-efficacy, neuroticism; and environmental factors such as lack of information about changes, lack of social, organizational and government supports are the major sources of stress.

Besides large volume of studies about the etiology of stress, there were also many studies on demographic data relating to stress. The most common variables for study are sex, teaching classes, marital status, teaching experiences, education, professional and religious backgrounds. These variables together with satisfaction with income were also examined in this study. In the studies of burnout among human service professionals including teachers by Maslach et al. (1996), results showed that female staff had higher emotional exhaustion than male colleagues. Mendes (2003) examined the relationship between emotional intelligence and teacher burnout on 49 credentialed secondary teachers and found that with more experience, teachers were better at identifying emotions. Lau et al. (2005) investigated the relationship between teachers' demographic variables and burnout in Hong Kong using the C-MBI on 1797 respondents from 45 secondary schools. Gender differences were found in all three burnout syndromes, and teachers who were younger, unmarried, without religious beliefs, less experienced, without finishing professional training and of junior rank were more consistently burned-out. Whereas age was the strongest predictor for emotional exhaustion and depersonalization, teachers' rank is the best predictor for personal accomplishment. In addition, Li et al. (2007) explored the relationship between teachers' sense of teaching efficacy and job burnout by using the Teachers' Sense of Teaching Efficacy Scale and the C-MBI which were administered to 247 secondary school teachers. Results indicated that teachers' length of teaching and marital status have significant effects on their personal teaching efficacy, depersonalization and reduced personal accomplishment.

However, there were also some inconsistent findings relating to relationship among the demographic variables and burnout. For instance, Holloman (1999) examined what personal and school-related variables were associated with 383 first-year school teacher burnout. No statically significant differences were found between areas of burnout and the variables of: gender, age and marital status. Likewise, the study by Zhao and Bi (2003) in a sample of 190 secondary school teachers in Chinese Mainland showed that there was no difference between the genders in the three burnout syndromes. A meta-analysis conducted by Weng (2005) in 35 US research studies with K-12 teacher populations concluded that all of the variables of human characteristics included in the demographics of the selected studies did not have very strong predictive correlations with burnout. That means a single factor, such as gender, cannot be used to predict if a person would be more than others susceptible to three dimensions of burnout.

Other studies also showed that burnout was associated with adverse health outcomes (Lee and Ashforth 1996; Maslach and Goldberg 1998). A recent study by Bian and Fan (2006) investigating the status of teacher's stress of 1012 middle school teachers and the relationship between stress and mental health in Chinese Mainland showed that the stress and the total score of Symptom Check List 90 (SCL-90) had positive correlation. It is suggested to improve the teacher's mental health by reducing their stress. Wang and Guo (2007) studied the relationship among occupational stress, teacher burnout and mental health state of 564 primary and middle school teachers in Chinese Mainland. Questionnaires about occupation stress, teacher burnout and SCL-90 Scale were used. Results showed that there were significant correlation among occupational stress, teacher burnout and mental health. There is a high level of occupational stress, teacher burnout and low level of mental health. In managing the burnout situation, social problem solving ability has been viewed as one of the coping strategies to stress in promoting personal well being. Many studies 
supported that higher social problem solving ability related to higher psychological wellbeing and general health (Dreer et al. 2005; Paul-Odouard 2006; Siu and Shek 2005a). Problem solving training in the workplace has been conducted to help employees to reduce distress (Ayres and Malouff 2007; Malouff et al. 2007). However, when Huey (2007) examined whether social problem solving ability moderates the effects of occupational stress on burnout, results indicated that though social problem solving ability significantly correlated with all three dimensions of burnout, more effective problem solving may not buffer against burnout under higher levels of occupational stress. Since no previous study regarding these areas is conducted in Macau, one of the aims of this study is to examine the relation of burnout situation to social problem solving and total well being.

With reference to Macau, though there were some studies on teacher stress, most studies adopted a survey approach with self-developed questionnaire which is difficult to be used for international comparison. Furthermore, little is known about the consequences of increasing stress. Education and Youth Affairs Bureau (2001) conducted a telephone survey on 450 school teachers of all primary and secondary education sectors out of 3194. Results showed that $43.0 \%$ of respondents felt stressful at work and $47.5 \%$ expressed frequent fatigue at school work. Coping strategies recommended included reducing teaching sessions, providing more free time for school teachers and gaining cooperation from parents. Another postal survey conducted by a teacher professional association on 717 school teachers indicated that $61.4 \%$ of respondents perceived their work as "exhausted work". The ranking of difficulties at work listed were lack of learning motivation of students, heavy workload and too many students per class (Macau Chinese Education Association 2002). Ng (2002) carried out a comparison study about teacher's time spent on 400 school teachers each on Beijing, Shanghai, Taipei, Hong Kong and Macau. Results showed that both teachers in Hong Kong and Macau were overloaded, working hours were double than those of the teachers of other three cities and time spent mostly were in class teaching, preparation for teaching and marking assignments. A study by Liu (2005) showed that when compared with Shanghai, Macau lacked approximately one-third of school teachers suggesting school teachers in Macau bear the workload of three teachers as compared with the workload of school teachers in Shanghai.

Since there is a paucity of empirical studies of teacher stress locally, and the relation of burnout with problem solving ability and personal well being is never studied in Macau, the present study can contribute to the understanding in these areas which can help design the stress reduction program as a means of coping strategy. Specifically, this study aims to explore the burnout situation of teachers in two schools in Macau and to investigate any differences made of demographic characteristics. The relation of burnout on social problem solving and the total well being of teachers is also examined. In this study, three questions are raised in the attempt to find out the burnout situation among the teachers in these two schools: (1) What is the level of different burnout syndromes of teachers in these two schools? (2) Do demographic characteristics make a difference in burnout situation? (3) Will the burnout situation relate to their social problem solving and total well being?

\section{Method}

\subsection{Participants and Procedures}

Ethical approval was obtained from the research review board of the Kiang Wu Nursing College in Macau. All the teachers of one primary and one secondary school were invited 
to participate in the study. The self administered questionnaires were sent to individual teachers and collected back by the respective school general offices. Voluntary bases and strict confidentiality were ensured. Verbal consents were obtained. Totally 138 teachers participated in the study. Seventy were from the primary school and 64 from the secondary school and four did not indicate. Seventy-six percent of participants were female and $23.19 \%$ were male. The age ranged from 21 to over 51 whereas the range from 26 to 30 and over 51 occupied more than $20.00 \%$ of the participants respectively. Regarding teaching experiences, $39.86 \%$ was under 5 years; however, $26.09 \%$ were over 21 years. The majority of them were with professional training status (83.33\%). More than half of them $55.80 \%$ were married. Nearly seventy percent of participants were mostly satisfied with their salary. Most of them $66.67 \%$ were without religious belief.

\subsection{Instruments}

\subsubsection{Chinese Maslach Burnout Inventory (C-MBI)}

The MBI was developed by Maslach and Jackson (1981) and the Chinese version was translated by Lau et al. (2005). The MBI consists of three dimensions with 22 items. They are: emotional exhaustion (EE) 9 items, depersonalization (DP) 5 items and reduced personal accomplishment (PA) 8 items. Maslach et al. (1996) reported the reliability coefficients of 0.90 for EE, 0.79 for DP and 0.71 for PA. The alpha coefficients of the present study for the three C-MBI were 0.85 for $\mathrm{EE}, 0.76$ for $\mathrm{DP}$ and 0.83 for PA. The participants were asked to rate the items on a 7-point Likert scale ranging from 0 (never) to 6(every day). The higher score in emotion exhaustion shows the feeling of drain and tiredness. The higher score in depersonalization indicates treating students as impersonal objects, while lower score in reduced personal accomplishment demonstrates feeling of inefficiency, ineffectiveness and inadequacy. The normative scores provided by Maslach et al. (1996) for teachers were used in assessing the levels of burnout: 27 or higher on EE; 14 or higher on DP; and 30 or lower on PA.

\subsubsection{Chinese Social Problem-Solving Inventory Revised (C-SPSI-R)}

The 52 item SPSI-R was developed by D'Zurilla et al. (1996) and the Chinese version with its short form was translated and developed by Siu and Shek (2005b). The C-SPSI-R consists of five dimensions with 25 items. They are: Negative problem orientation (NPO) 5 items, Rational problem solving (RPS) 5 items, Positive problem orientation (PPO) 5 items, Avoidance scale (AS) 6 items and Impulsiveness/Careless scale (ICS) 4 items. The Chinese version was found to be internally consistent with Cronbach's alpha for the 5 subscales varied from 0.65 to 0.88 and temporally stable over time with test-retest reliability for the 5 subscales as indicated by Pearson's $r$ varied from 0.48 to 0.79 . (Siu and Shek 2005b). The alpha coefficients of the present study for the 5 subscales varied from 0.68 to 0.83 . Studies show that SPSI-R is a liable and valid assessment instrument for research and clinical purposes to assess social problem-solving abilities and deficits (Chang and D'Zurilla 1996; D'Zurilla et al. 1996; Siu and Shek 2005a). The participants were asked to rate on a 5-point Likert scale ranging from (0) "Not at all true of me" to (4) "Extremely true of me". The NPO, AS and ICS consist of negative items indicating a dysfunctional or inhibitive orientation toward problem solving, while RPS and PPO consist of positive items indicating a constructive orientation. However the items are presented in a random order. A total score can be obtained by adding up the total positive scales (PPO 
and RPS) with the reverse scores of negative scales (NPO, AS and ICS). The higher the score indicates the better ability in problem solving.

\subsubsection{The Body-Mind-Spirit Well-Being Inventory (BMSWBI)}

The 56 item BMSWBI was developed in Chinese by Ng et al. (2005) to assess holistic health and comprised four scales: Physical distress 14 items, Daily functioning 10 items, Affect 19 items and Spirituality 13 items. The four scales have high reliability in their study, with alpha coefficients ranging from 0.87 to 0.92 , and concurrent validity. The alpha coefficients of the present study for the four scales ranged from 0.89 to 0.93 . The participants were asked to rate on an 11-point (or a 10-point) scale, with scores ranging from 0 (or 1) to 10; negative items were scored in reverse direction. The higher score in Physical distress shows the worse condition in health. On the contrary the higher scores in Daily functioning, Affect and Spirituality indicate the better condition in health. The scores of all items can be added up together to form a total score. The higher the total score indicates the better the overall well-being.

\section{Results}

\subsection{MBI Score of Participants}

To study the level of different burnout syndromes of the participants, the means and standard deviations of the three burnout syndromes were calculated. The mean scores for emotional exhaustion, depersonalization and personal accomplishment are 22.09 $(\mathrm{SD}=10.49), 6.70(\mathrm{SD}=5.86)$ and $31.36(\mathrm{SD}=9.01)$, respectively. The results are shown in Table 1. According to Maslach et al. (1996), scores of the MBI subscales are considered high if they fall into the upper third portion of the normative distribution, average if they fill into the middle third and low if they fall into the lower third. Overall, participants scored average in emotional exhaustion and personal accomplishment but low in depersonalization subscales. However, when using the normative scores provided in assessing the high level of burnout, some participants were experiencing high level of burnout. Thirty-four percent of the participants suffered from high level of emotional exhaustion with score 27 or higher, while $12.3 \%$ of the participants exhibited high level of depersonalization with score 14 or higher. In addition, $44.2 \%$ of the participants experienced lack of personal accomplishment with score 30 or lower. Finally, $4.3 \%$ (i.e., six persons) met the criteria of "highly burnout" with scores high on emotional exhaustion and depersonalization and low on personal accomplishment. Whereas $18.8 \%$ (i.e., 26 persons) suffered from quite serious condition with scores high on emotional exhaustion and low on personal accomplishment.

Table 1 Mean scores and standard deviations of the MBI subscales of Macau teachers

\begin{tabular}{lllrr}
\hline Maslach burnout inventory subcales & $n$ & Number of items & Mean & SD \\
\hline Emotional exhaustion & 138 & 9 & 22.09 & 10.49 \\
Depersonalization & 138 & 5 & 6.70 & 5.86 \\
Personal accomplishment & 138 & 8 & 31.36 & 9.01 \\
\hline
\end{tabular}


Table 2 Comparison of the categorization of MBI scores of Macau school teachers $(n=138)$ with secondary school teachers of Hong Kong $(n=1797)$ and school teachers of US $(n=4163)$
$E E$ emotional exhaustion,

$D P$ depersonalization,

$P A$ personal accomplishment

\begin{tabular}{lccc}
\hline MBI subscales & \multicolumn{3}{l}{ Range of experienced burnout } \\
\cline { 2 - 3 } & $\begin{array}{l}\text { Low } \\
\text { (lower third) }\end{array}$ & $\begin{array}{l}\text { Average } \\
\text { (middle third) }\end{array}$ & $\begin{array}{l}\text { High } \\
\text { (upper third) }\end{array}$ \\
\hline Macau teachers (Primary and secondary) & \\
EE & $<17$ & $18-26$ & $>27$ \\
DP & $<4$ & $5-7$ & $>8$ \\
PA & $>35$ & $34-28$ & $<27$ \\
Hong Kong teachers $($ grades $7-13)$ & & $>27$ \\
EE & $<17$ & $18-26$ & $>8$ \\
DP & $<3$ & $4-7$ & $<30$ \\
PA & $>38$ & $37-31$ & \\
US teachers (grades K-12) & & $>27$ \\
EE & $<16$ & $17-26$ & $>30$ \\
DP & $<8$ & $9-13$ & \\
PA & $>37$ & $36-31$ & \\
\hline
\end{tabular}

In order to help generate more insights into the burnout situation of Macau, data of the present study is compared with data from Hong Kong teachers (Lau et al. 2005) and North American teachers (Maslach et al. 1996). Adopting the method proposed by Maslach et al. (1996) for computing the cut-off points for the high, average and low range of the MBI subscales, the cut-off points for the Macau data were then calculated. When comparing the three MBI scores of Macau teachers with Hong Kong teachers, it is found that the ranges of all scores are similar to each other. When comparing the three MBI scores of Macau teachers with North American teachers, it is found that the ranges for emotional exhaustion scores and personal accomplishment scores from both places are similar to each other while the range of depersonalization scores of Macau teachers is much lower than that of the North American teachers. The results are presented in Table 2.

\subsection{Teacher Burnout and Demographic Characteristics}

One way ANOVA test was used to study the effects of demographic variables with teacher burnout. The demographic variables including the participants' gender, teaching class level, age, marital status, teaching experience, education background, professional qualification, satisfaction with income and religious belief were regarded as independent variables while the three burnout syndromes as dependent variables. Scheffe's test was conducted to examine group differences for those independent variables with two or more levels. The results are shown in Table 3.

No significant differences were found in all three burnout syndromes in gender, teaching class, professional qualification and religious belief while significant differences were found in some burnout syndromes in age, marital status, teaching experience, education background and satisfaction with income. Age was found to be one of the demographic variables that was related to burnout. Significant differences were found in emotional exhaustion and depersonalization. Younger teachers tended to show greater burnout syndromes than their older colleagues. According to Scheffe's procedure $(p<0.05)$, teachers below the age of 30 were more burned-out than those at the age of more than 31 and teachers at the age between 30 and 50 showed greater burnout syndrome than those above 
Table 3 ANOVA of the MBI subscales by the demographic variables of Macau school teachers

\begin{tabular}{|c|c|c|c|c|c|c|c|c|c|c|}
\hline \multirow[t]{2}{*}{ Variables } & \multirow[t]{2}{*}{$N$} & \multicolumn{3}{|c|}{ Emotional exhaustion } & \multicolumn{3}{|c|}{ Depersonalization } & \multicolumn{3}{|c|}{ Personal accomplishment } \\
\hline & & $M$ & SD & $F$ & $M$ & SD & $F$ & $M$ & SD & $F$ \\
\hline \multicolumn{11}{|l|}{ Sex } \\
\hline Male & 31 & 22.16 & 12.23 & 0.002 & 8.35 & 7.36 & 2.49 & 33.10 & 9.10 & 1.02 \\
\hline Female & 100 & 22.07 & 10.17 & & 6.47 & 5.28 & & 31.24 & 8.92 & \\
\hline \multicolumn{11}{|l|}{ Class } \\
\hline Primary & 66 & 22.32 & 11.42 & 0.008 & 7.16 & 6.01 & 0.21 & 31.66 & 9.36 & 0.001 \\
\hline Secondary & 62 & 22.48 & 9.43 & & 6.69 & 5.58 & & 31.60 & 8.59 & \\
\hline \multicolumn{11}{|l|}{ Age } \\
\hline Below 30 & 49 & 25.57 & 8.95 & $4.83 * *$ & 8.40 & 5.67 & $3.54 *$ & 30.81 & 7.81 & 1.68 \\
\hline $30-50$ & 58 & 20.48 & 10.8 & & 6.50 & 6.48 & & 30.95 & 9.83 & \\
\hline above 50 & 25 & 18.68 & 811.57 & & 4.85 & 3.87 & & 34.52 & 9.76 & \\
\hline \multicolumn{11}{|l|}{ Marital status } \\
\hline Single & 58 & 25.40 & 9.59 & $10.33 * *$ & 8.30 & 6.01 & $6.03 *$ & 32.33 & 7.55 & 0.55 \\
\hline Married & 73 & 19.66 & 10.57 & & 5.82 & 5.58 & & 31.16 & 9.74 & \\
\hline \multicolumn{11}{|c|}{ Teaching experience } \\
\hline Below 10 year & 66 & 23.85 & 9.46 & $3.84 *$ & 7.31 & 5.73 & 1.55 & 31.58 & 8.10 & 0.90 \\
\hline $10-20$ & 33 & 22.67 & 11.35 & & 7.52 & 6.91 & & 30.10 & 9.57 & \\
\hline Over 20 & 33 & 17.76 & 10.97 & & 5.35 & 4.79 & & 33.06 & 10.06 & \\
\hline \multicolumn{11}{|c|}{ Education background } \\
\hline Diploma & 61 & 20.02 & 10.58 & $4.16^{*}$ & 5.74 & 5.48 & $4.35^{*}$ & 32.25 & 9.97 & 0.63 \\
\hline Degree & 71 & 23.76 & 10.47 & & 7.83 & 6.04 & & 31.00 & 8.08 & \\
\hline \multicolumn{11}{|c|}{ Professional qualification } \\
\hline No & 14 & 25.07 & 8.80 & 1.26 & 7.30 & 5.03 & 0.05 & 32.55 & 6.86 & 0.09 \\
\hline Yes & 114 & 21.6 & 10.92 & & 6.91 & 6.00 & & 31.72 & 9.10 & \\
\hline \multicolumn{11}{|l|}{ Income } \\
\hline Satisfied & 95 & 20.81 & 10.02 & $5.63^{*}$ & 6.05 & 4.91 & $8.92 * *$ & 32.41 & 9.17 & 2.23 \\
\hline Dissatisfied & 33 & 25.82 & 11.60 & & 9.48 & 7.59 & & 29.76 & 7.98 & \\
\hline \multicolumn{11}{|l|}{ Religious belief } \\
\hline No & 87 & 22.85 & 10.82 & 0.08 & 7.38 & 6.07 & 1.06 & 31.08 & 8.85 & 1.11 \\
\hline Yes & 41 & 21.05 & 10.28 & & 6.24 & 5.38 & & 32.90 & 9.38 & \\
\hline
\end{tabular}

$N$ number of subjects, $M$ mean, $S D$ standard deviation, $F F$-value

$* p<0.05, * * p<0.01$

50. Teaching experience was another demographic variable related to burnout. Teachers with less than 10 year experiences were significantly more burn-out in emotional exhaustion than those working with over 20 year of experiences. Furthermore, teachers who were single, with better education background and dissatisfied with income were found consistently more burned-out in emotional exhaustion and depersonalization.

\subsection{Relation of Burnout with Problem Solving and Total Well-Being}

With the Pearson correlation test, both the C-SPSI and BMSWBI were found to be significantly negative correlated with emotional exhaustion and depersonalization and 
Table 4 Correlation of BMSWBI and MBI subscales, Correlation of C-SPSI and MBI subscales

\begin{tabular}{lllll}
\hline & & $\begin{array}{l}\text { Emotional } \\
\text { exhaustion }\end{array}$ & Depersonalization & $\begin{array}{l}\text { Personal } \\
\text { accomplishment }\end{array}$ \\
\hline BMSWBI and MBI subscales & & & $.232^{*}$ \\
BMSWBI $\quad \begin{array}{l}\text { Pearson correlation } \\
\text { Sig. (2-tailed) }\end{array}$ & $-.626^{*}$ & $-.459^{*}$ & 124 \\
& $N$ & 124 & 124 & $.492^{*}$ \\
C-SPSI and MBI subscales & Pearson correlation \\
C-SPSI & Sig. (2-tailed) & $-.463^{*}$ & $-.462^{*}$ & 138 \\
& $N$ & 138 & 138 & \\
\hline
\end{tabular}

$N$ Number of subjects, $* p<0.05$

positive correlated with personal achievement indicating that the more burned-out participants were the worse their problem solving ability and personal total well being would be. The results are presented in Table 4.

\section{Discussion}

\subsection{The Burnout Situation of Macau School Teachers}

It was found that Macau school teachers on the whole experienced moderate levels of emotional exhaustion, low levels of depersonalization, and moderate levels of personal accomplishment. However, many participants did suffer some psychological distress. Beyond six persons were found to be highly burned-out, twenty-six persons were demonstrating high levels of emotional exhaustion coupled with high levels of reduced personal accomplishment which would be problematic to the participants, their clients and the schools. These situations coincide with the findings of some local studies that school teachers in Macau are facing increasing stress (Education and Youth Affairs Bureau 2001; Macau Chinese Education Association 2002). Interventions are needed to assist them to prevent burnout.

When comparing the mean scores of the present study with the normative data of the Hong Kong teachers (Lau et al. 2005), we found that both teachers of Macau and Hong Kong scored in the average range of burnout in emotional exhaustion, depersonalization and personal accomplishment. These findings coincide with other comparison study between Macau and Hong Kong school teachers that both cities are facing the same problems (Ng 2002). When comparing the mean scores of the Macau data with the normative data of the teaching professionals of the North America (Maslach et al. 1996), Macau school teachers scored lower in depersonalization subscales than that of the North American teachers. These results coincided with the findings of Lau's study (Lau et al. 2005). As suggested, the expectation of the teacher role was different between the East and the West. The traditional role of teachers in the Chinese culture to treat their students in a strict and disciplined approach might be one of the reasons why both Macau and Hong Kong school teachers scored in depersonalization much lower than that of the North American teachers (Lau et al. 2005). Teachers in Macau and Hong Kong might not see themselves dehumanizing or indifferent to their students by being severe and controlling. 
On the contrary, they might perceive it as an effective way to train their students with a good will.

\subsection{Demographic Differences of Teacher Burnout}

Regarding gender differences, previous studies yielded inconsistent findings. In some western studies, Byrne $(1991,1999)$ found that female teachers reported greater burnout than male teachers among elementary and university educators in emotional exhaustion. On the other hand, no significant gender differences were found in emotional exhaustion in other studies (Maslach and Jackson 1986; Mills and Heubner 1998). In some eastern studies, Lau et al. (2005) found gender differences of the three burnout dimensions among teachers in Hong Kong secondary schools. Male teachers reported more depersonalization while female teachers experienced more emotional exhaustion and reduced personal accomplishment. On the other hand, no significant gender differences were found in the three burnout syndromes in a sample of 190 secondary school teachers in China Mainland. The results of the current study found no significant gender differences of each of the three burnout dimensions which provided evidence to support the inconsistent findings. Differences in the local context may be one explanation which requires further study.

Relating to teaching class, the present study found no difference in burnout situation between the primary and secondary school teachers which is inconsistent with previous studies such as the study by Anderson and Iwanicks (1984) that high school teachers were found to be more burned-out than teachers from elementary schools. There may be several possible reasons for this finding. First, Macau has been ruled by Portugal for more than 200 years before it is handed over to Mainland China in 1999. The values of enjoyment of life and living in a non-competitive environment of a small city like Macau are very much emphasized. Since competition is not encouraged, there is no public examination in both primary and secondary schools after graduation for promotion to the senior grade for studying. Therefore, no stress will be exerted to both primary and secondary school teachers to obtain a good performance of their students in the public examinations. Second, education is well supported by the government in Macau. There is a 15-year free education system from kindergarten to secondary schools. Even in local universities, there are quite a number of sponsorships and awards granted to those who are interested in further studying. Pressure to both students and teachers are less when compared with those countries that are short of support from the government. Third, in traditional Chinese culture, teaching is a respectable profession no matter teacher teaches in primary or secondary school.

With respect to professional qualification, the present finding is also inconsistent with the study of Lau et al. (2005) that teachers without professional training status were more burned-out than those with professional status. One possible reason is that 12 out of 14 participants without professional training status are university graduates who are capable to fulfill the requirement of professional training and pass the required examination organized by the government without much difficulty. Another reason may be due to the small number of participants without professional training status which cannot show the differences by statistical means.

With reference to religious belief, the present finding that no difference in burnout situation between teachers with and without religious belief is also found to be inconsistent with the study by Lau et al. (2005) that teachers with religious belief expressed significantly greater scores in personal accomplishment. One possible explanation is that religious commitment is not high in Macau. Data showed that $79.3 \%$ of Macau's population professes the religion of Buddhism, over 5.0\% are Roman Catholic and more than $1.0 \%$ are 
Protestants (US Consulate General 2007). Whereas in Hong Kong, only $43.0 \%$ of population participates in some form of religious practice and $9.6 \%$ are Christians including Catholics and Protestants (US Consulate General 2008). Though more people are with religious belief in Macau, as shown by Lui (2003) in a study of the local religion in Hong Kong and Macau, specifically referring to the folk religion including a mixture of Buddhism, Taoism and Confucianism, people will only seek a deity who can help them most in their particular area of difficulty. Furthermore, study by Luk and Shek (2006) indicated that only high levels of religious involvement (indexed by higher frequencies of church attendance) were generally related to psychological well being.

When the demographic variables with significant differences are concerned, the findings of the present study are in line with other studies that younger teachers have reported more emotional exhaustion and higher depersonalization (Maslach and Jackson 1981; Lau et al. 2005). It is understandable that young teachers are lacking life experiences in managing people including students, parents, colleagues and supervisors and they are more prone to job stress. When considering marital status, single persons were found to be more emotionally exhausted and depersonalizing than married persons, which were consistent with the findings by (De Heus and Diekstra 1999) and Li et al. (2007). Though divorce is not uncommon nowadays, marriage is still expected to be a lifelong commitment for a stable and enduring relationship which helps married persons to be emotionally supported to one and the other and kept healthy when facing life crisis such as job stress (Tischler 2007). Regarding teaching experiences, teachers with less teaching experience showed higher level of burnout in the emotional dimension, which are in line with the studies conducted by Mo (1991) and Lau et al. (2005) in Hong Kong. Study by Mendes (2003) also supported that with more experience, teachers are better at identifying emotions which helps cope with stress. With respect to education background, those with higher education background expressed more emotional exhaustion and higher depersonalization which may be due to their higher expectation to oneself and to their students. Concerning satisfaction with income, those with dissatisfaction felt more emotionally exhausted and depersonalizing. One possible explanation is that the feeling of unfairness in what they contribute to their work will affect their morale which in turn will increase their emotion drainage.

As far as these demographic variables with significant differences are concerned, some burnout syndromes are found in school teachers who are younger, single, with less teaching experience, higher education background and dissatisfied with income. These findings can provide useful information to the school administrators in developing strategies to combat burnout. Beyond organizing induction program and mentor system for those new teachers who are young and green in the teaching profession, a more challenging in-service training program should also be developed to provide continuing education and enhancement to those who are more academically prepared. Furthermore, a caring atmosphere and a strong team spirit have to be cultivated to provide better social support to those who are single and to those dissatisfied with income to be diverted from only monetary incentives.

\subsection{Relation of Burnout with Problem Solving and Total Well-Being}

A significant correlation between burnout and personal well-being is in line with other studies (Bian and Fan 2006; Wang and Guo 2007) that high level of burnout may affect one's health in terms of low level of holistic health. Likewise, all three dimensions of burnout were found to be significantly correlated with social problem solving ability indicating that burnout may affect one's problem solving ability. Siu and Shek (2005a) studied the relations between social problem solving and emotional well-being and found 
that high levels of social problem solving were related to better emotional well-being. Study by Huey (2007) also showed that social problem solving ability significantly correlated with all three dimensions of burnout and suggested that evaluating one's social problem solving ability in addition to occupational stress may allow for better identification of those who may be at risk of burnout. Seeing that burnout is related to social problem solving and personal well-being, it is important to design a well structured and evaluative stress reduction program for those who may be more prone to burnout. However, such intervention program is scarce in Macau.

\subsection{Limitations of the Study}

There are some limitations of the study. First, the sample size was small; therefore the power of analyses was reduced. However, it still met the minimal numbers for meaningful analysis. Second, the samples came from two schools; the results cannot be generalized to the general population. Third, the cutoff scores of the MBI into high, average and low range of subscales are arbitrary. However, it is still an acceptable way for comparison between different samples. Finally, the management culture of the two schools were not explored which might affect the understanding of its influences to the participants in coping with stress and burnout.

\section{Conclusion}

The study found that teachers of two schools in Macau experienced relatively average burnout which coincided with the findings in Hong Kong. These findings added on the data base for comparison internationally among the Chinese population. The findings identified those with greater degree of burnout, to whom more attention should be paid. Furthermore, it once again supported that level of burnout may affect one's problem solving ability and holistic health. Finally, the study also contributed to the limited literature on the quality of life of teachers in Macau.

Open Access This article is distributed under the terms of the Creative Commons Attribution Noncommercial License which permits any noncommercial use, distribution, and reproduction in any medium, provided the original author(s) and source are credited.

\section{References}

Anderson, M. B., \& Iwanicki, E. F. (1984). Teacher motivation and its relationship to burnout. Educational Administration Quarterly, 20, 94-132.

Ayres, J., \& Malouff, J. M. (2007). Problem-solving training to help workers increase positive affect, job satisfaction, and life satisfaction. European Journal of Work and Organizational Psychology, 16(3), 279-294.

Bian, B. Q., \& Fan, F. M. (2006). Teachers stress and mental health in middle school. Chinese Mental Health Journal, 20, 48-50.

Byrne, B. M. (1991). Burnout: investigating the impact of background variables for elementary, intermediate, secondary, and university educators: Teaching and teacher education. An International Journal of Research, 7, 197-209.

Byrne, B. M. (1999). The nomological network of teacher burnout: A literature review and empirically validated model. In R. Vandenberghe \& A. M. Huberman (Eds.), Understanding and preventing teacher burnout (pp. 15-37). Cambridge: Cambridge university Press. 
Chang, E. C., \& D’Zurilla, T. J. (1996). Relations between problem orientation and optimism, pessimism, and trait affectivity: A construct validation study. Behaviour Research and Therapy, 34, 185-194.

D'Zurilla, T. J., Nezu, A. M., \& Maydeu-Olivares, A. (1996). Manual for the social problem-solving inventory-revised (SPSI-R). NY, Tonawanda: Multi-Health Systems, Inc.

De Heus, P., \& Diekstra, R. F. W. (1999). Do teachers burn out more easily? A comparison of teachers with other social professions on work stress and burnout symptoms. In R. Vandenberghe \& A. M. Huberman (Eds.), Understanding and preventing teacher burnout (pp. 269-284). Cambridge: Cambridge university Press.

Dreer, L. E., Elliott, T. R., Fletcher, D. C., \& Swanson, M. (2005). Social problem solving abilities and psychological adjustment of persons in low vision rehabilitation. Rehabilitation Psychology, 50(3), 232-238.

Dworkin, A. G. (1997). Coping with reform, the intermix of teacher morale, teacher burnout, and teacher accountability. In B. J. Biddle, T. L. Good, \& I. F. Goodson (Eds.), International handbook of teachers and teaching (pp. 459-498). Dordrecht/Boston/London: Kluwer Academic Publishers.

Education and Youth Affairs Bureau. (2008a). The general situation of non-tertiary education in Macau in academic year 2007/2008. Retrieved February 20, 2009, from Macau Special Administrative Region, Macau Web site: http://www.dsej.gov.mo/ webdsej/www/einter_dsej_page.php.

Education and Youth Affairs Bureau. (2008b). Academic and professional qualification: Law 15/96/M- the general rules for teaching personnel. Retrieved March 2, 2009, from Macau Special Administrative Region, Macau Web site: http://www.dsej.gov.mo/ webdsej/www/edulaw/basic/d1596mc.htm.

Education and Youth Affairs Bureau. (2001). A survey of teacher work stress 2001. Macau: Macau Special Administrative Region. in Chinese.

Freudenberger, H. J. (1974). Staff burn-out. Journal of Social Issues, 30, 159-165.

US Consulate General (2008). General information: Background note, Hong Kong. Retrieved September 2, 2008, from http://hongkong.usconsulate.gov/ushk_2008.html.

Holloman, H. L. (1999). Factors related to burnout in first-year teachers in South Carolina. Dissertation, University of South Carolina.

Huey, S. T. (2007). Occupational stress, social problem solving, and burnout among mental health professionals in HIV/AIDS care. Dissertation, University of Drexel.

Lau, P. S. Y., Yuen, M. T., \& Chan, R. M. C. (2005). Do demographic characteristics make a difference to burnout among Hong Kong secondary school teachers? Social Indicators Research, 71, 491-516.

Lee, R. T., \& Ashforth, B. E. (1996). A meta-analytic examination of the correlates of the three dimensions of job burnout. Journal of Applied Psychology, 81, 123-133.

Li, Y. X., Yang, X., \& Shen, J. L. (2007). The relationship between teachers' sense of teaching efficacy and job burnout. Psychological Science (China), 30, 952-954.

Liu, S. B. (2005). Teachers' strategies in facing stress. (Paper presented the conference on Enhancing Teachers' Professionalism for Chinese Community, Hong Kong). Retrieved September 3, 2008. from http://cpc.emb.org.hk/conference/download/liu.doc.

Lui, T. S. (2003). A nameless but active religion: An anthropologist's view of local religion in Hong Kong and Macau. In D. L. Overmyer (Ed.), Religion in China today. New York: Cambridge University Press. 72 .

Luk, A. L., \& Shek, D. T. L. (2006). Changes in Chinese discharged chronic mental patients attending a psychiatric rehabilitation program with holistic care elements: A quasi-experimental study. TSW Holistic Health and Medicine, 1, 71-83. doi:10.1100/tswhhm.2006.26.

Macau Chinese Education Association. (2002). Teaching job make you exhausted. Macau Education, 2, 51-63. (in Chinese).

Malouff, J., Thorsteinsson, E., \& Schutte, N. (2007). The efficacy of problem solving therapy in reducing mental and physical health problems: A meta-analysis. Clinical Psychology Review, 27, 46-57.

Maslach, C. (1976). Burned-out. Human Behavior, 5, 7-22.

Maslach, C., \& Goldberg, J. (1998). Prevention of burnout: New perspectives. Applied and Preventive Psychology, 7, 63-74.

Maslach, C., \& Jackson, S. E. (1981). The measurement of experienced burnout. Journal of Occupational Behavior, 2, 99-113.

Maslach, C., \& Jackson, S. E. (1986). Maslach burnout inventory manual (2nd ed.). CA: Consulting Psychologists Press.

Maslach, C., Jackson, S. E., \& Leiter, M. (1996). Maslach burnout inventory manual (3rd ed.). CA: Consulting Psychologists Press.

Mendes, E. J. (2003). The relationship between emotional intelligence and occupational burnout in secondary school teachers. Dissertation, University of Walden. 
Mills, L. B., \& Heubner, E. S. (1998). A prospective study of personality characteristics, occupational stressors, and burnout among school psychology practitioners. Journal of School Psychology, 36, 103120.

Mo, K. W. (1991). Teacher burnout: Relation with stress, personality, and social support. Education Journal, 19, 3-12.

$\mathrm{Ng}, \mathrm{K} . \mathrm{T}$. (2002). A comparison study on teacher's activity time and characteristics between Macau and Beijing. Teacher Journal, 5, 42-49. (in Chinese).

Ng, S. M., Yau, J. K. Y., Chan, C. L. W., Chan, C. H. Y., \& Ho, D. Y. F. (2005). The measurement of bodymind-spirit well-being toward multidimensionality and transcultural applicability. Social Work in Health Care, 41, 33-52.

Paul-Odouard, R. (2006). Emotional intelligence, social problem solving, and demographics as predictors of well-being in women with multiple roles. Dissertation, University of Adelphi.

Sarros, A. M., \& Sarros, J. C. (1990). How burned out are our teachers? A cross-cultural study. Australian Journal of Education, 34, 145-152.

Siu, A. M. H., \& Shek, D. T. L. (2005a). Relations between social problem solving and indicators of interpersonal and family well-being among Chinese adolescents in Hong Kong. Social Indicators Research, 71(1), 517-539.

Siu, A. M. H., \& Shek, D. T. L. (2005b). The Chinese social problem solving inventory: Some initial results on reliability and validity. Journal of Clinical Psychology, 61, 347-360.

Tischler, H. L. (2007). Introduction to sociology (9th ed.). CA: Thomson, Wadsworth. p306.

US Consulate General. (2007). U.S. and Macau, religious demography. Retrieved September 2, 2008, from http://hongkong.usconsulate.gov/usmo_hr_2007091401.html.

Wang, W. Z., \& Guo, L. Y. (2007). An investigation on occupational stress, teacher burnout and mental health state of primary and middle schools teachers and the relations among them. Chinese Journal of Clinical Psychology, 15, 146-148. (in Chinese).

Weng, C. H. (2005). Meta-analysis of teacher burnout in public schools in the United States. Dissertation, University of South Dakota.

Xu, F., Ji, F., \& Chao, Q. (2004). The development of job burnout questionnaire in primary and secondary teachers. Chinese Journal of Clinical Psychology, 12, 13-14. (in Chinese).

Zhang, J. (2007). A cultural look at information and communication technologies in Eastern education. Education Technology Research and Development, 55, 301-314. doi:10.1007/s114230079040.

Zhao, Y., \& Bi, C. (2003). Job burnout and the factors related to it among middle school teachers. Psychological Development and Education, 1, 80-84. (in Chinese). 